\title{
Reliable Designing of Capacitated Logistics Network with Multi Configuration Structure under Disruptions: A Hybrid Heuristic Based Sample Average Approximation Algorithm
}

\author{
D. Shishebori ${ }^{1}$
}

\begin{abstract}
We consider the reliable multi configuration capacitated logistics network design problem (RMCLNDP) with system disruptions, concerned with facilities locating, transportation links constructing, and also allocating their limited capacities to the customers in order to satisfy their demands with a minimum expected total cost (including locating costs, link constructing costs, as well as expected transshipment costs in usual and disruption conditions). The motivating application of this class of problem is in capacitated logistics network design with multi configuration (including multi-product, multi-vehicle, and multi-type link) regarding system disruptions simultaneously. The problem is modelled as a mixed integer program. Also, a hybrid heuristic algorithm is proposed. The algorithm, as an efficient approach, is a hybridization of sample average approximation, the LP relaxation, and a two stage decomposing heuristic. The results of a detailed comprehensive computational analysis are also reported. Computational experiments illustrate that the provided algorithm is able to substantially outperform the integer programming approach in terms of both finding and verifying the efficient optimal (or near optimal) solutions at reasonable processing times.
\end{abstract}

Keywords: Multi-product, Multi-type transportation links, Multi-vehicles, Multi configuration, Capacitated logistic network design, Disruptions, Reliability, Two stage decomposing heuristic, LP relaxation, Sample average approximation (SAA).

Manuscript was received on 23/12/2015, revised on 05/12/2017 and accepted for publication on 30/11/2017.

\section{Introduction}

Due to the manufacturing competitive world of the 21 st century, nowadays, the supply chain network design (SCND) issue and its related topics have a special importance in optimization. Because this issue is a significant subject that can play a key role in the reduction of various costs (such as costs of location, construction, operation, production, and transportation) as well as in increasing the efficiency of production and service systems.

The past studies demonstrate that a variety of SCND problems have been solved by the development of several mathematical programming models. Some reviews have mentioned the models, algorithms and applications; see (Daskin et al., 2005) [13], (Meixell and Gargeya, 2005) [37], (Vahdani et al., 2012) [62], (Hatefi et al., 2014) [23], (Cardona-Valdes et al., 2014) [10], (Vahdani, 2015) [67], and (Keyvanshokooh et al., 2016) [31].

\footnotetext{
${ }^{1}$ Department of Industrial Engineering, Yazd University, Yazd, Iran, Email: Shishebori@ Yazd.ac.ir.
} 
Since SCND is known as a long term strategic decision problem, so considering various practical factors can help in getting more efficient solutions of the problem under study. Disruption is known as a key factor in this context. Recently, this topic in SCND has become a considerable issue for most companies in the trade globalization world.

In one view, several potential sources of disruptions threaten the supply chains. In general, disruptions are unplanned and unanticipated events that can cause several perturbations in the normal flow of products and materials within a supply chain. The disruption at one member of a supply chain (SC) can make a significant influence over the whole chain. SCs are threatened by several potential sources of disruptions. Some of them are external sources (e.g., natural disaster, terrorist attack, power outage, and supplier discontinuity) and some of them are internal sources (e.g., industrial accident, labor strike). Although these disruptive events may possibly cause short-term facility eventualities, they may also lead to not only severe operational consequences, such as higher transshipment costs, order delays, inventory shortages, loss of market shares, and so on, but also increased negative financial effects; see (Peng et al., 2011) [43], and (Keyvanshokooh et al., 2013) [30].

Here, we study the problem of designing a capacitated SCN, subject to system disruptions (including facility disruptions and transshipment link disruptions). Once such an SC structure is constructed, it will probably be very hard and expensive to change the design. Therefore, it surely is much worthed to develop an SC that achieves permanence and efficiency in the presence of all kinds of disruptions.

The significant contributions of this study are arranged as follows: (i) the reliable capacitated SCND problem with a multi configuration structure is studied regarding facility disruptions and transshipment link disruptions simultaneously, (ii) a hybrid heuristic algorithm, a hybridization of obtaining sample average approximation (SAA), LP relaxation, and a two stage decomposing heuristic, is proposed to solve the problem by near-optimal solutions in reasonable times. To the best of our knowledge, such study has not been made in the literature.

The remainder of this paper is organized as follows. Section 2 provides a relatively comprehensive literature review in three main streams. Section 3 presents the problem definition and the proposed mathematical programming model. Then, in Section 4, at first, a customized SAA for the RMCLNDP is proposed and then, a hybrid heuristic algorithm, a hybridization of SAA, the relaxation, and a two stage decomposing heuristic, is described in detail. In Section 5, analysis of solving the test problems, computational results and discussions about the efficiency of the proposed HHBSAA algorithm are presented. Finally, conclusions and guidelines for future research are provided in Section 6.

\section{Literature Review}

In the SCND and logistics literature, system disruption is known as an special issue of supply uncertainty. The disruptions are introduced as casual events leading to a supplier or other components of the supply chain to halt functioning, either completely or partially, for usually a partial amount of time. Several robust strategies and approaches have been proposed to mitigate the effects of supply chain disruptions and improve the efficiency of SC and its logistics at disruption conditions. For more study, the reader is referred to the review by (Snyder and Daskin, 2006) [57]), (Snyder et al., 2012) [55], and (Vahdani et al., 2012) [62].

In order to clear up the description of our contribution, three main streams of the literature are reviewed that may be interesting for comparison: (i) SCND subject to facility disruptions, (ii) SCND 
subject to transportation link disruptions, and (iii) capacitated SCND. It is noted that in this study, the system disruptions are defined as facility disruptions and transshipment link disruptions.

\subsection{Facility Disruptions}

The literature related to facility disruptions demonstrates that, at first, (Drezner, 1987) [14] studied facility location with unreliable suppliers by formulating some mathematical models. They considered the unreliable $p$-median and $(p, q)$-center location problems, in which a probability of becoming inactive was specified for each facility. In the following studies, Snyder (2003) [54], Snyder and Daskin (2005) [56], and Snyder and Daskin (2007) [58] considered the reliable $p$-median and fixed charge problems due to several level assignments with the unreliable candidate sites. Several approaches have been considered to address the facility disruptions within mathematical formulations; see Snyder et al., (2012) [55].

Scenario is a known approach related to uncertain conditions. In most stochastic programming models, there are disruption scenarios in which all (or sometimes a sample) of the disruption scenarios are enumerated. This approach is simple and tangible, but, once the number of facilities and subsequently the problem size grows, it becomes insupportable. Snyder and Daskin (2006) [57] proposed some mathematical models using this approach. Peng et al. (2011) [43] considered the effect of system failure conditions in logistic network design problems with facility disruptions as several scenarios. Moreover, Jabbarzadeh et al. (2012) [26] formulated an SC design problem with the distribution centers having partial or complete disruptions. Aydin and Murat (2013) [4] studied the capacitated reliable facility location problem (CRFLP) regarding facility disruptions as scenarios. They proposed an efficient algorithm as a hybridization of particle swarm intelligence (PSO) and sample average approximation (SAA) methods. Garcia-Herreros et al. (2014) [21] developed a two-stage stochastic program in order to design resilient SCs in which the distribution centers are subject to the risk of disruptions at each potential location. The problem is concerned with selecting distribution center locations, determining storage capacities for multiple commodities, and making suitable distribution strategies in scenarios that explain disruptions at potential distribution centers. Cardona-Valdes et al. (2014) [10] formulated a two-stage stochastic problem in order to minimize the total cost and the total service time simultaneously, for designing a two echelon production-distribution network with multiple manufacturing plants, distribution centers and a set of candidate warehouses. They proposed a scenario based approach in order to consider the impacts of the uncertainty. Ivanov et al. (2014) [25] formulated a multi-period and multi-commodity distribution (re)planning problem for a multi-stage centralized upstream network with structured dynamic conditions. Their approach considers several execution scenarios and proposes some efficient suggestions on (re)planning in the case of system failures. Also, the graph of structural reliability identifies the optimistic and pessimistic scenarios.

Probability distribution terms is another approach in which the probability distributions of the uncertain conditions are known to decision makers and the related terms can be applied in proposing the mathematical model. Berman et al. (2007) [8] studied a $p$-median problem in which the facility disruptions have the probability with a similar value as suggested by Snyder and Daskin (2005) [56]. They focused on structural properties and special cases of their non-linear model and presented two formulations for the reliable facility location problem (RFLP) model of Snyder and Daskin (2005) [56] with site-specific disruption probabilities. One model applies a scenario approach within a stochastic programming framework, while the other one involves computing the expected travel costs endogenously using highly non-linear multiplicative terms in a manner similar to Berman et al. (2007) [8]. They developed a heuristic based on the sample average approximation of Kleywegt et al. (2002) [32] for the first model and two greedy-adding-type heuristics for the latter. Computational results 
showed that one of the greedy-adding methods outperforms the other two heuristics. Also, for the problem with site-specific disruption probabilities, a non-linear formulation was developed by Cui et al. (2010) [12]. They reformulated their model using the reformulation-linearization technique (RLT), resulting in a model that is both linear in the decision variables and polynomial in the problem size. They also introduced a continuum approximation (CA) model that requires simulation and regression, but may be solved in closed form to allow for managerial insights. In another study, Aboolian et al. (2012) [1] reformulated the MINLP model of Cui et al. (2010) [12] by an approximate model in which the probability that a customer was assigned to a given facility was calculated assuming that all closer facilities were open.

Comparing the two scenario and probabilistic approaches, it can be observed that in the probabilistic approach, for each event, a probability distribution is considered and its related probability is proposed in the calculations. Although it tends to provide a better perspective of the problem, applying this approach generally leads to a considerable complexity of the problem so that finding an appropriate and effective response for the problem is faced with several difficulties. In contrast, the scenario approach assign a specified numerical value to each event and this value is used in calculations. Although applying these approaches may turn to reduce the application of the obtained solution, by using the SAA approach, the solving procedure and the complexity of the mathematical formulation will be significantly reduced even in large sizes, and subsequently, efficient solutions can be achieved at the reasonable times.

Other approaches are considered to address the facility disruptions. Lim et al. (2009) [35] presented that wrong estimation of the disruption probability can have some considerable negative effects on the obtained solution of the facility location problem under random facility disruptions. Cui et al. (2010) [12] studied another CA model, in which disruptions are assumed to be geographically correlated. They introduced closed-form approximations for the total cost and the individual cost components and demonstrated that these were quite accurate. Berman (2010) [9] considered a continuous version of the median problem with unreliable facilities (MPUF) model in which two unreliable facilities, with correlated disruptions, are to be located on a line segment. They developed closed-form solutions for the problems and performed parametric analysis to describe how the optimal locations and costs change when the disruption characteristics change. O'Hanley and Church (2011) [40] formulated a robust locating of the facility location-interdiction covering model. Shishebori et al. (2013) [48] and Shishebori and Jabal Ameli (2013a,b) [49, 50] considered facility disruptions as a constraint for the maximum allowable disruption cost of the system. They proposed a MINLP model for the problem and investigated it by a case study. Haldar et al. (2014) [22] developed an efficient fuzzy group decision making approach due to a fuzzy technique for strategic supplier selection in a fuzzy environment regarding a disaster scenario. Recently, Azizi et al. (2014) [5] proposed a mathematical model in order to study building of hub-and-spoke network systems under the risk of hub disruption. Their model assumed that once a hub stops normal operations, the entire demand initially served by the hub is handled by a backup facility. The objective function of the model minimizes the weighted sum of transportation cost in regular situation and the expected transportation cost following a hub failure. Fattahi et al. (2015) [18] developed different mixed-integer linear programming (MILP) models for designing of centralised and decentralized supply chains by using two-stage stochastic programming. They investigated a multiple period replenishment problem based on the $(\mathrm{s}, \mathrm{S})$ policy for these supply chain models. To deal with demand uncertainty, scenarios were generated using the Latin Hypercube Sampling method and their number was reduced by a scenario reduction technique. To solve this problem, they proposed an evolutionary strategy (ES) and an imperialist competitive algorithm (ICA) to find near optimal solutions. 


\subsection{Link Disruptions}

Some studies related to link disruptions were performed. Most of them, in the literature, extensively studied the design of survivable telecommunication networks (Soni, 2000) [60]. Ferris (2000) [20] proposed a robust two-stage flow model where the plan specifies the value of flow to be sent to the static optimal route and the value of rerouted flow in presence of the probability of some disruptions. Waller (2002) [69] presented some algorithms for the online shortest path problems regarding capacitated link-cost dependencies. In their study, disruptions were considered by using online-recourse models where once real-time information becomes available, the remaining path till the destination is re-evaluated. Sever et al. (2013)[47] considered networks in which the travel time of the transshipment links can increase, because the links are subject to several disruptions and are subsequently vulnerable. They considered some predetermined link disruption probabilities for recovering from or getting into a disruption. For more reviews, we refer to Soni (2000) [60], Klibi (2009) [33], Vahdani et al. (2013) [63, 64], Esmaeilikia et al. (2014) [17], and Vahdani and Mohammadi (2015) [68].

As a remarkable point, it should be mentioned that reliable communication networks can still work suitably when some partial system failures happen in some components. However, the amount of the flow and the total cost of these networks significantly differ from those in supply chains.

\subsection{Capacitated Network Design}

Capacity is known as another significant factor that plays a critical role in SCND. Several studies were done regarding the SC limited capacity. Mahajan et al. (2002) [36] analyzed an SC consisting of uncapacitated/capacitated suppliers for distributing two independent commodities through multiple retailers and studied the problem by means of game theory. Jemai and Karaesmen (2007) [28] considered the framework of a Nash game for a two-stage SC consisting of a capacitated supplier and a retailer. (Sitompul et al. 2008) [53] studied the effect of the safety stock placement strategy for an $n$-stage capacitated serial SC as a shortest path problem and presented an efficient approach with the objective of maintaining the essential overall service level at the lowest cost. Toktas-Palut (2011) [61] investigated a decentralized two-stage SC with multi independent capacitated suppliers. They modeled the problem as an M/M/1 make-to-stock queuing network in order to coordinate the inventory policies of the suppliers in the SC. In their study, the end-customer demand follows a Poisson distribution probability, and the service times of the suppliers are exponentially distributed. Nepal et al. (2012) [39] investigated a three echelon supply chain with some capacity restrictions and step-changes in supply consumption rate regarding the life-cycle demand phases. Duan and Liao (2013) [15] proposed a simulation-based optimization approach in order to determine the nearoptimal supply chain replenishment policies in presence of various demands and control strategies for a capacitated supply chain. They examined a capacitated single distributor-multi retailer supply chain system in detail. Shishebori et al. (2013) [48] considered facility disruptions via a constraint on the maximum allowable disruption cost of the system in the context of a facility location-network design problem with disruptions. They proposed a MINLP model for the problem and illustrated it by a case study. Shishebori et al. (2014) [51] consider a similar facility location-network design problem and proposed an LP-based heuristic to solve it. Recently, Rahmaniani and Ghaderi (2015) [44] developed some simple and efficient meta-heuristics based on the variable neighborhood search method in order to solve the CFLNDP effectively. Moreover, other related studies dealt with the capacitated supply chain in different capacitated conditions (e.g., Jemai and Karaesmen (2007) [28], Hennet and Arda (2008) [24], Vahdani et al. (2013) [63, 64], and Vahdani et al. (2014) [65]). 


\subsection{Supply Chain Network Design with Multi-Configuration}

Considering several possible aspects of the proposed problem can lead to more practical solutions. This can help to decision makers to have several alternatives for the proposed SCND and logistics. The several possible aspects can include multi-product, multi-type link, and multi-vehicle. In this paper, we refer to them by multi-configuration.

Several studies were done at the SCND and logistics with multi-configuration structure. Chen and Lee (2004) [11] formulated a multi-product multi-stage multi-period model with multiple incompatible goals of a multi-echelon SCN as a MINLP, in which the fuzzy sets were considered to explain the uncertainties involved in market demands and product prices. Park et al. (2007) [41] formulated a multi-period multi-product SC model, including supplier, manufacturer, and distribution center to minimize the total cost. You and Grossmann (2008) [70] proposed the optimization of a bicriteria multi-echelon supply chain in presence of demand uncertainty with the objectives of maximizing the net present value and minimizing the expected lead time. Ferrio and Wassick (2008) [19] developed a MILP model for redesigning and optimizing a multi-product chemical supply network, including production sites, an arbitrary number of DCs, and customers. Also, El-Sayed et al. (2010) [16] considered maximizing the total expected profit of a multi-period three-echelon forward-reverse logistics network under demand uncertainty in the forward direction and deterministic customer demand in the reverse direction. Mirzapour Al-E-Hashem et al. (2011) [38] modelled a multi-site, multi-period, multi-product three echelon SC in presence of uncertainties of cost parameters and demand changes. They solved the proposed bi-objective problem as a singleobjective mixed integer programming (MIP) problem through an LP-metric method. Amrani et al. (2011) [3] proposed a MIP model for a multi-commodity production-distribution network with alternative facility configuration and solved it by a variable neighborhood search (VNS) method. Bashiri et al. (2012) [7] formulated a new multi-product mathematical model for a multi-echelon network with strategic and tactical planning and different time resolution decisions. Jamshidi et al. (2012) [27] modelled a bi-objective multi-echelon SCN design problem in which several transshipment alternatives at each level of the chain were considered with different costs and a limited capacity.(Karimi-Nasab et al. (2013) [29] proposed a multi-objective approach in order to determine the distribution policy for a wholesaler. In their approach, the wholesaler distributes supplementary nutrition to a set of local distribution centers positioned around the wholesaler, geographically. Their approach optimizes selling price, carrying cost, batch size and services level of multi-items for each local distribution centre in every planning period. Badri et al. (2013) [6] presented a mathematical technique based on the Lagrangian relaxation method for solving a multi-commodity SCND model with different time resolutions for strategic and tactical decisions. The objective function maximizes the total net income over time. Moreover, Song et al. (2014) [59] modelled a manufacturing SC problem with multiple suppliers. Their proposed SC is subject to several simultaneous uncertainties such as uncertain material supplies, stochastic production times, and random customer demands. Recently, Pasandideh et al. (2014) [42] proposed a mathematical model for a bi-objective optimization of a multi-product multi-period three-echelon SC network problem considering several system uncertainties. The goal is to minimize the total production costs, supply and warehouse costs, transportation costs, inventory costs, and shortage costs such that the expected and the variance of the total cost are simultaneously minimized. Sarrafha et al. (2014) [45] studied an SCND including suppliers, factories, DCs, and retailers. They proposed a multi-period structure such that a flow-shop scheduling model in the manufacturing part of the $\mathrm{SC}$, and also, the shortage in the form of backorder in each period were integrated. Their main goals are minimizing the total SC costs as well as minimizing the average tardiness of the product to DCs. Shishebori and Yousefi (2015) [52] studied a robust and reliable medical service (MS) center location network design problem, which simultaneously takes uncertain parameters, system disruptions, and investment budget constraint into 
account. Their programming model is formulated based on an efficient robust optimization approach to protect the network against uncertainty. In the literature, another approach to the parameter uncertainty makes use of fuzzy theory. In several decision making situations, high degree of uncertainties, with fuzziness aspect, are involved in the data set. Fuzzy set theory provides a framework for handling the uncertainties of this type.

\subsection{Research Gap and Our Contribution}

Some existing works studied the SCND and logistics with facility disruptions regarding link disruptions separately. However, most methods did not consider the capacity (including facilities and transportation links). But, in some manufacturing industries, there are some capacitated SC and logistics systems in which a variety of disruptions (failures) may occur. The most obvious examples are SC of different spare parts, food products manufacturing, petrochemicals, etc.

Here, we investigate the problem of designing a capacitated SC network, consisting of suppliers, DCs, and demand nodes as well as some transportation links. These are considered as potentials and it should be decided which potential nodes and links should be built. It is noted that modifying the SCND and its related logistics will be very difficult and costly.

\section{Problem Definition and Formulation}

The working conditions of the RMCLNDP can be described as follows. Suppose that there is an SC network $\mathrm{GG}=(G, A)$. Let $G_{S}, G_{T}$, and $G_{D}$ signify the sets of supply, transshipment, and demand nodes, respectively. Also, let $G_{0}$ be defined as the set of all supply and transshipment nodes $\left(G_{0}=\right.$ $G_{S} \cup G_{T}$ ), in which the nodes can have open/close final situation after decision making. The set $G_{0}$ can be called "facilities". All of the facilities and transportation links are capacitated and each has a maximum level (capacity) in order to give service to the demand nodes. Let $S$ be the set of scenarios, each of which specifies a set of facilities and transportation links that are simultaneously disrupted. Suppose $s=0$ as the nominal scenario in which no disruptions happen. The set $P$ illustrates several types of products that should be produced and transshipped to the demand nodes. The set $L$ presents different kinds of transshipment links. For example, for each link, it is assumed that three several quality levels (i.e., $|L|=3$ ), each of which is defined as follows: the dirt road (type 3 ), the paved road with low quality (type 2) and the paved road with the standard quality (type 1). As a remark, if a link with type 1 quality is constructed, its construction cost will be more than the other types, but its capacity is more and its transshipment cost is lower than the other types of road links (Rahmaniani and Ghaderi, 2015) [44]. Here, links (roads) with three quality types are defined; however, several quality types can be defined for the problem. The set $V$ shows several types of transportation vehicles. The best type of the vehicle has the highest cost of investment, but the lowest cost of transportation.

In order to avoid the infeasible situations, a penalty fee is ordained for the demands of nodes that cannot feasibly be met. It can be denoted that these demands are fulfilled from some outside suppliers as emergency facilities but with high transportation costs. Also, it can be interpreted that the demand of a node cannot be served if the penalty is smaller than the cost of serving the demand of the node. Here, this contingency is considered by supposing that $N_{S}$ involves an "emergency facility" such that it has no fixed cost and it is never disrupted, and also has infinite capacity. Obviously, in the optimal solution, it is always open and doesn't have any disruptions. For each transshipment link, from the emergency facility to other nodes, the unit transshipment cost is equal to the unmet-demand penalty fee. The sets and parameters of the problem are defined as follows. 
Sets:

$G$ : $\quad$ set of nodes $\left(G=G_{S} \cup G_{T} \cup G_{D}\right)$,

$G_{0}$ : set of "facilities" nodes $\left(G_{0}=G_{S} \cup G_{T}\right)$,

$P: \quad$ set of products $(P=1,2,3, \ldots)$

$L: \quad$ set of several quality types of transportation links $(L=1,2,3, \ldots)$

$V$ : set of several types of transportation vehicles $(V=1,2,3, \ldots)$

$A$ : set of potential transportation links

$S$ : set of scenarios; each scenario illustrates the facilities and also link disruptions

\section{Parameters:}

$f_{j}$ = fixed cost for locating of facility $j \in G_{0}$

$\pi^{s}=$ probability of happening of scenario $s \in S$

$c_{i j}{ }^{l}=$ construction cost of link $(i, j) \in A$ with quality type $l(l \in L)$

$t_{i j}{ }^{p l v}=$ unit transshipment cost of product $p(p \in P)$ on link $(i, j) \in A$ with quality type $l(l \in L)$ by vehicle $v(v \in V)$

$\gamma_{i j}{ }^{v}=$ investment cost of vehicle $v(v \in V)$ at link $(i, j) \in A$

$\lambda_{i j}{ }^{v}=$ capacity of vehicle $v(v \in V)$ at link $(i, j) \in A$ (in kilograms)

$\Gamma_{j}=$ capacity of facility at node $j \in N_{S}$ (according to processing time criterion)

$\varphi_{J}^{p}=$ processing time of product $p(p \in P)$ at node $j \in G_{S}$

$\Theta_{j}=$ capacity of facility at node $j \in N_{T}$ (in kilograms)

$\Pi_{i j}{ }^{l}=$ capacity of link $(i, j) \in A$ with quality type $l(l \in L)$ (in kilograms)

$\psi^{p}=$ weight of product $p(p \in P)$

$U_{i j}^{\max }=$ maximum number of vehicle types which can be used at link $(i, j) \in A$

$b_{J}^{p}=b_{J}^{p} \geq 0$ if $j \in G_{s}$, representing the supply of product $p(p \in P) ; b_{J}^{p}=0$, if $j \in G_{T}$, representing the transshipment of product $p(p \in P)$; and $b_{J}^{p} \leq 0$ if $j \in G_{D}$, representing the demand of product $p(p \in P)$

$$
\begin{aligned}
\Phi_{j}^{s} & = \begin{cases}1, & \text { if facility at node } j \in N_{0} \text { is disrupted in scenario } s \in S \\
0, & \text { otherwise }\end{cases} \\
\Omega_{i j}{ }^{s} & = \begin{cases}1, & \text { if link }(i, j) \in A \text { is disrupted in scenario } s \in S \\
0, & \text { otherwise }\end{cases} \\
\Delta_{i j}^{l v s} & = \begin{cases}1, & \text { if vehicle } v(v \in V) \text { at link }(i, j) \in A \text { with quality type } l(l \in L) \text { is disrupted in scenario } s \in S \\
0, & \text { otherwise }\end{cases}
\end{aligned}
$$

Although the $\Phi_{j}^{s}, \Omega_{i j}{ }^{s}$, and $\Delta_{i j}^{l v s}$ are defined as binary parameters, the proposed mathematical programming model may function well if these parameters are considered to be fractional, indicating partial disruptions.

\section{Decision variables:}

$$
\begin{gathered}
Z_{j}= \begin{cases}1, & \text { if a facility is located at node } j \in G_{0} \\
0, & \text { otherwise }\end{cases} \\
X_{i j}{ }^{l}= \begin{cases}1, & \text { if link }(i, j) \in A \text { is constructed with quality type } l(l \in L) \\
0, & \text { otherwise }\end{cases} \\
W_{i j}^{v}= \begin{cases}1, & \text { if vehicle } v(v \in V) \text { is established at link }(i, j) \in A \\
0, & \text { otherwise }\end{cases}
\end{gathered}
$$


$Y_{i j}^{p l v s}=$ amount of flow of product $p(p \in P)$ on $\operatorname{link}(i, j) \in A$ with quality type $l(l \in L)$ by vehicle $v(v \in V)$ in scenario $s \in S$

Therefore, we propose the following MIP model for the RMCLNDP:

$$
\begin{aligned}
& \min \quad E T C=\sum_{j \in G_{0}} f_{j} Z_{j}+\sum_{(i, j) \in A} c_{i j}^{l} X_{i j}^{l}+\sum_{v \in V} \sum_{(i, j) \in A} \gamma_{i j}^{v} W_{i j}^{v} \\
& +\sum_{p \in P} \sum_{l \in L} \sum_{v \in V} \sum_{(i, j) \in A} t_{i j}^{p l v} \sum_{s \in S} \pi_{s} Y_{i j}^{p l v s} \\
& \sum_{l \in L} \sum_{v \in V} \sum_{(j, i) \in A} Y_{j i}^{p l v s}-\sum_{l \in L} \sum_{v \in V} \sum_{(i, j) \in A} Y_{i j}^{p l v s} \leq b_{j}^{p}, \quad \forall j \in G_{S} ; \quad s \in S ; \quad p \in P \\
& \sum_{l \in L} \sum_{v \in V} \sum_{(j, i) \in A} Y_{j i}^{p l v s}-\sum_{l \in L} \sum_{v \in V} \sum_{(i, j) \in A} Y_{i j}^{p l v s}=0, \quad \forall j \in G_{T} ; \quad s \in S ; \quad p \in P \\
& \sum_{l \in L} \sum_{v \in V} \sum_{(j, i) \in A} Y_{j i}^{p l v s}-\sum_{l \in L} \sum_{v \in V} \sum_{(i, j) \in A} Y_{i j}^{p l v s}=b_{j}^{p}, \quad \forall j \in G_{D} ; \quad s \in S ; \quad p \in P \\
& \sum_{p \in P} \sum_{l \in L} \sum_{v \in V} \sum_{(i, j) \in A} \varphi_{j}^{p} Y_{i j}^{p l v s} \leq\left(1-\Phi_{j}^{s}\right) \Gamma_{j} Z_{j}, \quad \forall j \in G_{S} ; \quad s \in S \\
& \sum_{p \in P} \sum_{l \in L} \sum_{v \in V} \sum_{(i, j) \in A} \psi^{p} Y_{i j}^{p l v s} \leq\left(1-\Phi_{j}^{s}\right) \Theta_{j} Z_{j}, \quad \forall j \in G_{T} ; \quad s \in S \\
& \sum_{p \in P} \sum_{l \in L} \sum_{v \in V} \Theta_{j} Y_{i j}^{p l v} \leq\left[\left(1-\Omega_{i j}^{s}\right) \Pi_{i j}^{l} \sum_{l \in L}\left(X_{i j}^{l}+X_{j i}^{l}\right)\right], \quad \forall(j, i) \in A ; \quad s \in S \\
& \sum_{p \in P} \psi^{p} Y_{i j}^{p l v s} \leq\left[\left(1-\Delta_{i j}^{l v s}\right) W_{i j}^{v} \lambda_{i j}^{v}\right], \quad \forall(i, j) \in A ; l \in L ; v \in V ; s \in S \\
& \sum_{l \in L}\left(X_{i j}^{l}+X_{j i}^{l}\right) \leq 1, \quad \forall(i, j) \in A \\
& \sum_{v \in V} W_{i j}^{v} \leq U_{i j}^{\max }, \quad \forall(i, j) \in A \\
& W_{i j}^{v} \leq \sum_{l \in L}\left(X_{i j}^{l}+X_{j i}^{l}\right), \quad \forall(i, j) \in A ; \quad v \in V \\
& Z_{j} \in\{0,1\}, \quad \forall j \in G_{0} \\
& W_{i j}^{v} \in\{0,1\}, \quad \forall(i, j) \in A ; v \in V \\
& X_{i j}^{l} \in\{0,1\}, \forall(i, j) \in A ; l \in L \\
& Y_{i j}^{p l v s} \geq 0, \quad \forall(i, j) \in A ; p \in P ; l \in L ; v \in V ; s \in S .
\end{aligned}
$$

The objective function (1) minimizes the expected total costs (ETC), involving fixed location costs, link construction costs, vehicle establishment costs, and also the expected transshipment costs for all possible scenarios with respect to their probabilities. Constraints (2)-(4) are known as the flow conservation constraints. Constraints (2) emphasize that, for supply nodes, the flow out should be less than or equal to the supply. Also, constraints (3) enforce that the flow in should be to equal the flow out for transshipment nodes. Sequentially, for demand nodes, constraints (4) ensure that the flow in 
will be to equal the demand. It is noted that each of supplier, DCs and demand nodes can be applied as transshipment nodes, i.e., the demands can be imported and exported from each of the supplier, DCs and demand nodes. Constraints (5) ensure that the summation of processing time of products, produced by supply node $j \in G_{S}$, cannot exceed its total processing time capacity $\Gamma_{j}$ when it is opened (i.e. $Z_{j}=1$ ) and is fully functional in scenario $s \in S$, and prevent any flow when it is closed or disrupted. Also, constraints (6) guarantee that the summation of weights of the flow of products, transported by DC $j \in G_{T}$, does not exceed its capacity $\Theta_{j}$ when it is opened (i.e. $Z_{j}=1$ ) and is totally functional in scenario $s \in S$, and prevent any flow when it is disrupted or closed. Moreover, constraints (7) emphasize that the total flow through the link $(i, j) \in A$ does not exceed its capacity $\Pi_{i j}{ }^{l}$ when it is constructed (i.e., $X_{i j}{ }^{l}+X_{j i}{ }^{l}=1$ ) and is fully functional in scenario $s \in S$, and prevent any flow when it is disrupted or closed. Constraints (8) guarantee that the summation of weights of the flow, transshipped by vehicle $v$ through the link $(i, j) \in A$ with type $l$, does not exceed its capacity $\lambda_{i j}{ }^{v}$ when it is established (i.e., $W_{i j}{ }^{v}=1$ ) and is totally functional in scenario $s \in S$, and prevent any flow when it is closed or disrupted. Constraints (9) prevent links from being opened in both directions with several quality types at once. Constraints (10) limit the maximum number of vehicle type that can be applied through the link $(i, j) \in A$. Constraints (11) guarantee that the vehicle establishing at link $(i, j) \in A$ can be happed when the link, with at least one of the quality type, is opened. Constraints (12)-(14) declare the binary variables and finally, constraints (15) guarantee that the variable $Y_{i j}^{p l v s}$ will be non-negative.

Now, knowing that the RMCLNDP can be reformulated as an extended version of the $p$-LNDP, which is itself NP-hard, proposition 1 presents that RMCLNDP is NP-hard,.

Proposition 1. The RMCLNDP is NP-Hard.

Proof. In the $p$-LNDP, introduced by Peng et al. (2011) [43], let $p$, the desired robustness level, be equal to infinity; therefore, the $p$-robust constraint in the $p$-LNDP is inactive. Moreover, let $p_{s}=1$ for the nominal scenario $s=0$ and $p_{s}=0 \quad \forall s \in S \backslash\{0\}$. Also, assume that the disruption scenarios do not include any link disruptions and all of the links are uncapacitated. Moreover, assume that there is just only one kind of product which is produced and transshipped, there is only one quality type link can be opened, and there is only one type of vehicle that can be used in the links. Then, RMCLNDP reduces to $p$-LNDP, which is NP-hard Peng et al. (2011) [43]. Therefore, RMCLNDP is NP-hard.

\section{Solution Procedure}

Since the RMCLNDP is categorized as an NP-hard combinatorial optimization problem, even solving the medium-size test instances can be very hard with conventional methods. This practically intensifies specially when the number of scenarios considerably. In this section, we propose two solution methods. At first, the classical sample average approximation (SAA) method, is customized to solve the RMCLNDP. Then, our proposed HHBSAA algorithm is presented. In order to improve the solution quality and efficiency, the hybrid HHBSAA algorithm integrates a heuristic strategy inspired by LP-relaxation with the classical SAA method.

\subsection{Sample Average Approximation (SAA)}

One of the most efficient solution approaches to solve large size stochastic optimization problems is SAA method Ahmed and Shapiro (2002) [2], Kleywegt et al. (2002) [32], Schutz et al. (2009) [46], Aydin and Murat (2013) [4]. In a general view, the SAA method solves a sample of scenarios of the 
problem and accordingly, it approximates the objective function value of the stochastic program. Accordingly, the final solution is the best performing sample solution. In the following, the efficiency of the proposed hybrid procedure with that of SAA is considered and evaluated. The steps of the SAA method to solve the RMCLNDP are as follows.

\section{SAA method for RMCLNDP:}

Initialize: Generate $M$ independent random samples $(m=1,2, \ldots, M)$ with the scenario sets $N^{m}$, where $\left|N^{m}\right|=N$. Each sample $m$ consists of $N$ conceptions of independently and identically distributed random scenarios. Besides, a reference sample which is large enough is selected; e.g., $\left|N^{\prime}\right| \gg N$.

Step 1: For each sample $m$, solve the following optimization problem and save the sample optimal objective function value $\xi^{m}$ and the sample optimal solution $Z^{m}=\left\{Z_{j}^{m}\right\}_{\forall j \in G_{0}}$ :

SAA- RMCLNDP $(m)$ :

$$
\begin{aligned}
& \min \quad E T C=\sum_{j \in G_{0}} f_{j} Z_{j}^{m}+\sum_{(i, j) \in A} c_{i j}^{l} X_{i j}^{l m}+\sum_{v \in V} \sum_{(i, j) \in A} \gamma_{i j}^{v} W_{i j}^{v m} \\
& +\sum_{p \in P} \sum_{l \in L} \sum_{v \in V} \sum_{(i, j) \in A} t_{i j}^{p l v} \sum_{s \in N_{m}} \frac{1}{\left|N_{m}\right|} Y_{i j}^{p l v s m} \\
& \sum_{l \in L} \sum_{v \in V} \sum_{(j, i) \in A} Y_{j i}^{p l v s m}-\sum_{l \in L} \sum_{v \in V} \sum_{(j, i) \in A} Y_{i j}^{p l v s m} \leq b_{j}^{p}, \quad \forall j \in G_{S} ; \quad s m \in N_{m} ; \quad p \in P \\
& \sum_{l \in L} \sum_{v \in V} \sum_{(j, i) \in A} Y_{j i}^{p l v s m}-\sum_{l \in L} \sum_{v \in V} \sum_{(j, i) \in A} Y_{i j}^{p l v s m}=0, \quad \forall j \in G_{T} ; \quad s m \in N_{m} ; \quad p \in P \\
& \sum_{l \in L} \sum_{v \in V} \sum_{(j, i) \in A} Y_{j i}^{p l v s m}-\sum_{l \in L} \sum_{v \in V} \sum_{(j, i) \in A} Y_{i j}^{p l v s m}=b_{j}^{p}, \quad \forall j \in G_{D} ; \quad s m \in N_{m} ; \quad p \in P \\
& \sum_{p \in P} \sum_{l \in L} \sum_{v \in V} \sum_{(i, j) \in A} \varphi_{j}^{p} Y_{i j}^{p l v s m} \leq\left(1-\Phi_{j}^{s m}\right) \Gamma_{j} Z_{j}^{m}, \quad \forall j \in G_{S} ; \quad s m \in N_{m} \\
& \sum_{p \in P} \sum_{l \in L} \sum_{v \in V} \sum_{(i, j) \in A} \psi^{p} Y_{i j}^{p l v s m} \leq\left(1-\Phi_{j}^{s m}\right) \Theta_{j} Z_{j}^{m}, \quad \forall j \in G_{T} ; \quad s m \in N_{m} \\
& \sum_{p \in P} \sum_{l \in L} \sum_{v \in V} \sum_{(i, j) \in A} \Theta_{j} Y_{i j}^{p l v s m} \leq \\
& {\left[\left(1-\Omega_{i j}^{s m}\right) \Pi_{i j}^{l} \sum_{l \in L}\left(X_{i j}^{l m}+X_{j i}^{l m}\right)\right],} \\
& \forall(j, i) \in A ; \quad s m \in N_{m} \\
& \sum_{p \in P} \Theta^{p} Y_{i j}^{p l v s m} \leq\left[\left(1-\Delta_{i j}^{l v s}\right) W_{i j}^{v m} \lambda_{i j}^{v}\right], \quad \forall(i, j) \in A ; l \in L ; v \in V ; \quad s m \in N_{m} \\
& \sum_{l \in L}\left(X_{i j}^{l m}+X_{j i}^{l m}\right) \leq 1, \\
& \forall(i, j) \in A \\
& \sum_{v \in V} W_{i j}^{v m} \leq U_{i j}^{\max }, \\
& \forall(i, j) \in A \\
& W_{i j}^{v m} \leq \sum_{l \in L}\left(X_{i j}^{l m}+X_{j i}^{l m}\right), \\
& \forall(i, j) \in A \quad ; \quad v \in V
\end{aligned}
$$




$$
\begin{array}{ll}
Z_{j}^{m} \in\{0,1\}, & \forall j \in G_{0} \\
W_{i j}^{v m} \in\{0,1\}, & \forall(i, j) \in A ; \quad v \in V \\
X_{i j}^{l m} \in\{0,1\}, & \forall(i, j) \in A ; l \in L \\
Y_{i j}^{p l v s m} \geq 0, & \forall(i, j) \in A ; p \in P ; l \in L ; v \in V ; s m \in N_{m} .
\end{array}
$$

Step 2: Calculate the average $\bar{\xi}^{M}$ of the sample optimal objective function values obtained in step 1 as follows:

$$
\bar{\xi}^{M}=\frac{1}{M} \sum_{m=1}^{M} \xi^{m} .
$$

Step 3: Estimate the true objective function value $\hat{\xi}^{m}$ of the original problem to each sample's optimal solution. Solve the following optimization problem for each sample using the optimal first stage decisions $Z^{m}$ from Step 1:

$$
\begin{gathered}
\hat{v}^{m}=\min \\
\sum_{j \in G_{0}} f_{j} Z_{j}^{m}+\sum_{(i, j) \in A} c_{i j}^{l} X_{i j}^{l m}+\sum_{v \in V} \sum_{(i, j) \in A} \gamma_{i j}^{v} W_{i j}^{v m} \\
+\sum_{p \in P} \sum_{l \in L} \sum_{v \in V} \sum_{(i, j) \in A} t_{i j}^{p l v} \sum_{s \in N^{\prime}} \frac{1}{\left|N^{\prime}\right|} Y_{i j}^{p l v s m}
\end{gathered}
$$

subject to constraints (16) to (30), and using $N_{m} \equiv N^{\prime}$.

Step 4: Select the solution $Z^{m}$ with the best $\hat{\xi}^{m}$; i.e., $Z^{\mathrm{SAA}}=\arg \min _{\mathrm{m}=1, \ldots, \mathrm{M}} \hat{\xi}^{m}$, as the solution and $\xi^{S A A}=\min _{\mathrm{m}=1, \ldots, \mathrm{M}} \hat{\xi}^{m}$, as the solution value of SAA.

The optimal objective function value of the original problem RMCLNDP is denoted by $\xi^{*}$. Moreover, $\bar{\xi}^{M}$ illustrates an unbiased estimator of $\operatorname{Exp}[\xi]$, which is the expected optimal objective function value of sample problems. Since $\operatorname{Exp}[\xi] \leq \xi^{*}, \bar{\xi}^{M}$ can be known as a statistical lower bound on the $\xi^{*}$; see Ahmed and Shapiro (2002) [2]. While $\bar{\xi}^{M}$ cannot always be a lower bound on the $\xi^{*}$, it is beneficial to evaluate the quality of the solution value of SAA $\hat{\xi}^{S A A}$. The reference set $N^{\prime}$ is applied to estimate the objective function value of the sample problem solutions in the original problem RMCLNDP.

\subsection{Hybrid Heuristic Based SAA (HHBSAA) Algorithm}

The proposed algorithm, as an efficient approach, is a hybridization of the SAA, the LP relaxation, and a two stage decomposing heuristic. The stimulation for this hybridization arises from the last stage of the SAA method (Step 4) in which the best performing solution is selected and the rest is abandoned. However, this abandoning of $(M-1)$ sample solutions can lead to both a loss of precious sample knowledge and a loss of time spent in solving each sample's solution. We consider combining the implementation of the classical SAA procedure and the LP-relaxation in the context of the proposed heuristic and use all of the obtained information of the sample solutions together. This 
combined approach is worked as follows. At first, the SAA method is applied such that for each $m$, the LP-relaxation of the $S A A-R M C L N D P(m)$ is solved and the vector $Z^{m}$ is obtained. The elements of the vector $Z^{m}$ are not necessarily binary, because we solved the LP-relaxation of the $S A A$ $R M C L N D P$. Therefore, we have $M$ vector $Z$. A new vector $Z$ is established regarding the $M$ vector $Z$ such that the sum of all elements in one column are calculated and then, the non-zero elements in the summation of the $M$ vector $Z$ (called $Z^{\text {sum }}$ ) are changed to 1 at the new vector $Z\left(Z^{\text {new }}\right)$. Here, we explain this technique with an illustrative example. Assume that $M=7$ and $\left|G_{0}\right|=10$, that is, the vector $Z$ has 10 elements. By executing the SAA, 7 vectors $Z^{m}$ are obtained as shown in Fig. 1.

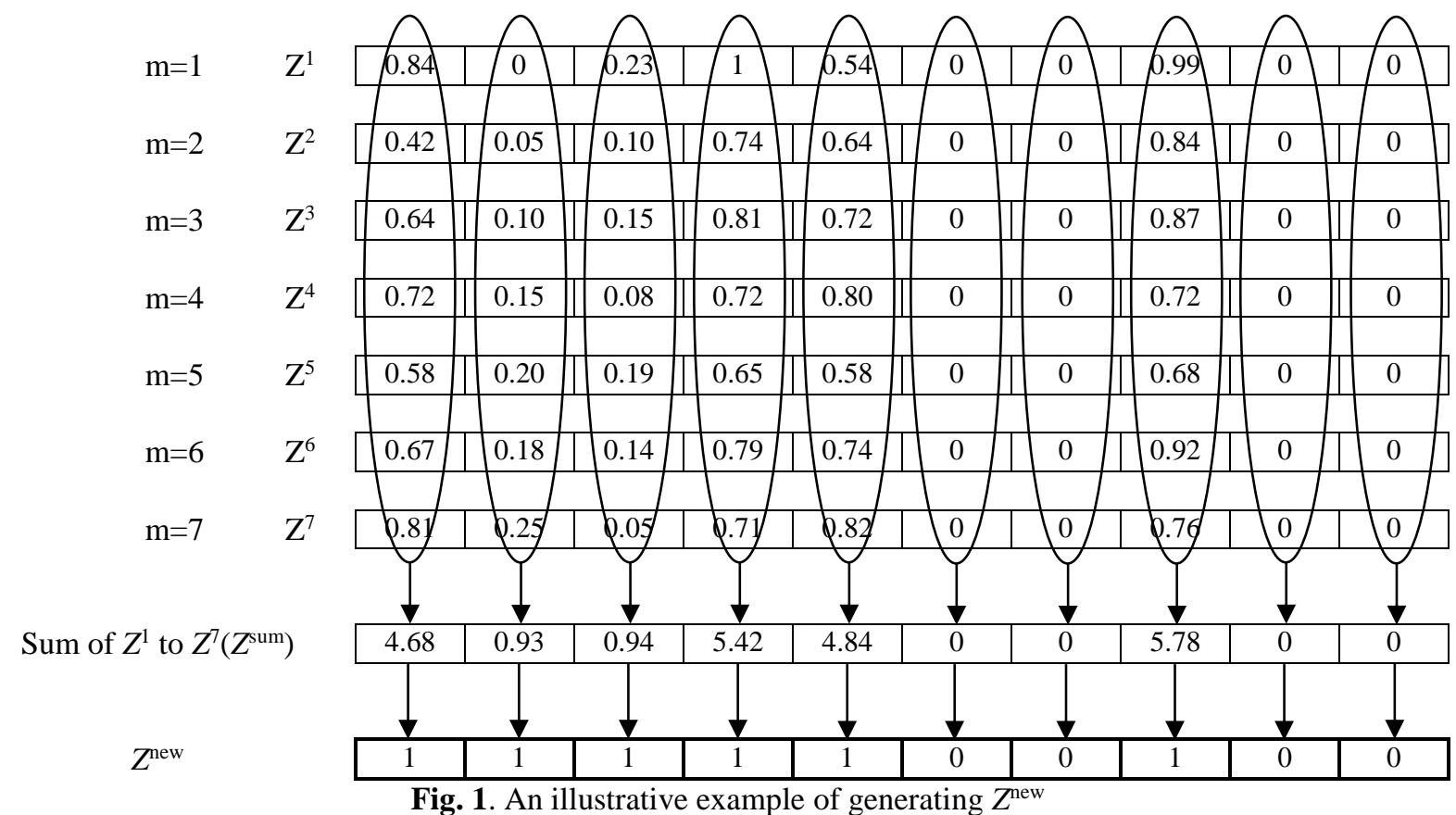

The $Z^{\text {new }}$ is obtained by combining the obtained information due to all the vectors $Z^{m}$ together, as presented in Fig. 2.

At the following, $Z^{\text {new }}$ is used as an input data (initial solution) in stage 2 of the algorithm (stage 2: Setting and solving using Fig. 3). In fact, the overall logic of the algorithm is organized such that the original problem is disintegrated into two sub-problems. At the first stage of the first sub-problem, the LP-relaxation of the $S A A-R M C L N D P$ is solved and $Z^{\text {new }}$ is obtained. Then, $Z^{\text {new }}$ is set as the input data matrix to the second stage and the main model (RMCLNDP) is solved by applying the input data matrix. Accordingly, the complexity of the model of RMCLNDP is reduced by fixing of some integer variables (i.e., vector Z). It is mentioned that HHBSAA solves the sample problems to optimality. However, since the samples are not related to all members of the scenario set, these optimal solutions are sub-optimal for the original problem, unless the reference sample set is equal to all of the scenarios (i.e., $N^{\prime} \equiv S$ ). The main steps of HHBSAA algorithm are presented in Fig. 2. 


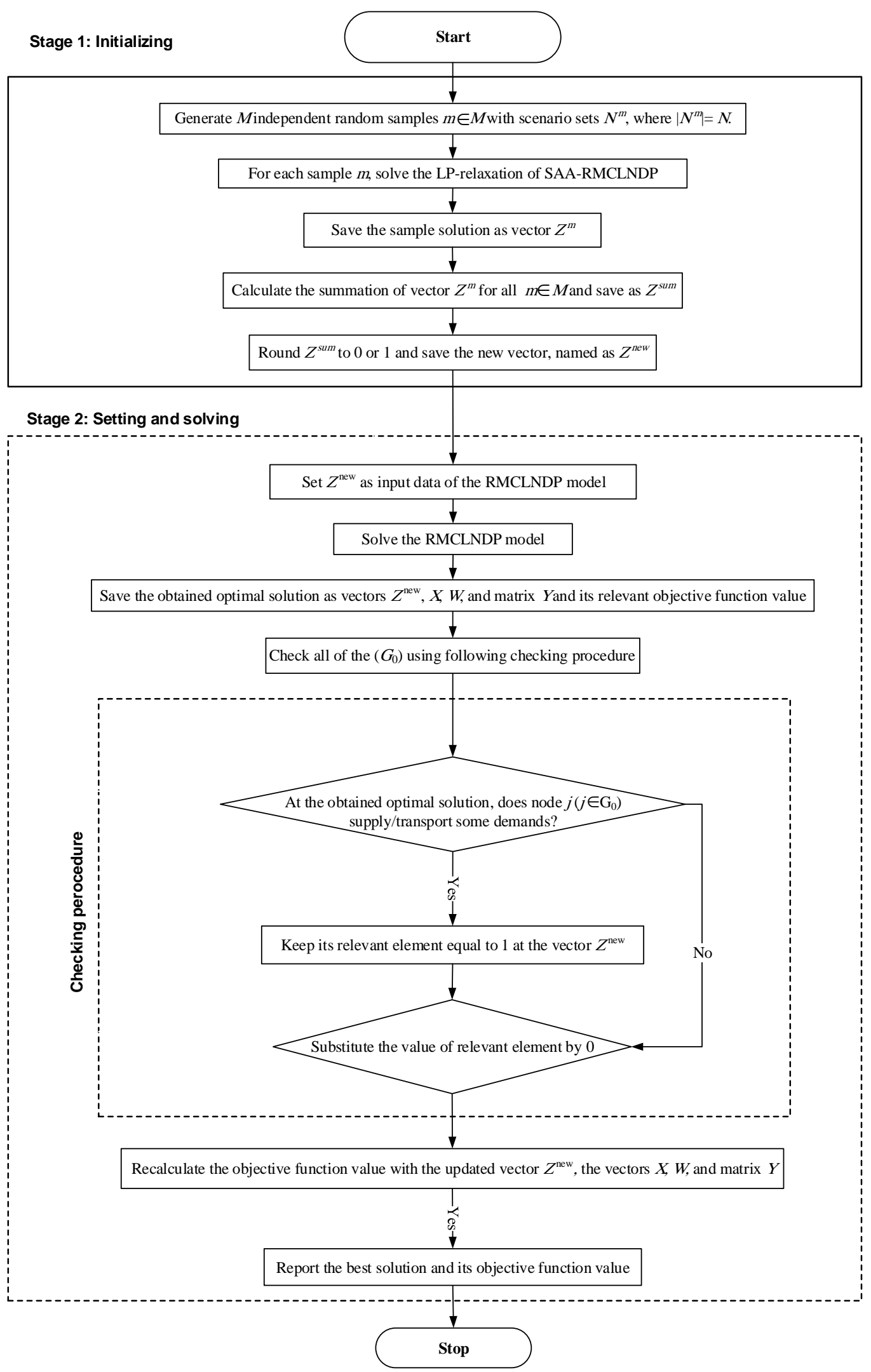

Figure. 2. The main procedure of the HHBSAA algorithm 


\section{Results and Discussions}

Numerous test data are generated and solved by the CPLEX 12.1 solver in the software package GAMS 23.5.1. Then, the proposed algorithms were coded in MATLAB R2011a. All computations are carried on a PC with Windows 7 professional, with $2.67 \mathrm{GHz}$ dual core processor and $4 \mathrm{~GB}$ of RAM. Also, the CPU solution time was limited to 3600 seconds for CPLEX solver and the proposed heuristic algorithms.

\subsection{Design of Experiments}

In order to demonstrate the efficiency of the proposed heuristic algorithms, several test problems were solved with different sizes of $M$ and $N$. The procedure for the random test problems generation is similar to that described in the literature (Peng et al., 2011) [43]. In more details, random test problems with different sizes were generated. The numbers of supply nodes, transshipment nodes and demand nodes were fixed to 2, 3, and 4 respectively. Also, the number of scenarios at $S$ is set 300 . The edge density was chosen from $25 \%, 35 \%$ and $55 \%$. At the beginning, the number of supplier, DCs and demand nodes was selected and accordingly the constructed transshipment links among nodes were specified by the edge density regarding the predetermined probability. The links between the "emergency" facility and the demand nodes were opened with probability 1.

The fixed costs $f_{j}$ of suppliers and of DCs were generated uniformly from [25000, 30000] and [5000, 10000], respectively. Also, the unit transshipment costs $t_{i j}^{p l v}$ were drawn uniformly from [1, 500]. The per-unit transshipment cost from the emergency facility to each demand node was named unmet-demand penalty and set to 1400 . At each demand node, the parameter $b_{j}^{p}$ is a negative number and illustrates the demand of product $p$. This was also generated uniformly from $[-110,-50]$. At each supply node, the supply $b_{j}^{p}$ was specified as follows. To provide a warranty for the feasibility of the model regarding most of the data sets, at first, the average necessary supply of product $p$, $\bar{s}$, was calculated by

$$
\bar{s}^{p}=\frac{\left|G_{D}\right|}{\left|G_{S}\right|} \cdot \bar{d}^{p},
$$

where $\bar{d}^{p}$ is the average demand of product $p$, which is set to 50, 60, and 70, respectively, in this case. Each $b_{J}^{p}$ was then generated uniformly from $\left[1 \bar{s}^{p}, 3 \bar{s}^{p}\right]$.

The capacity of a supply node $j, \Gamma_{j}$, is drawn uniformly from [1000, 2000], as minutes (according to the processing time criterion). Moreover, the capacity of each DC is specified equivalently by first calculating

$$
\bar{c}=\frac{\sum_{p \in P} \sum_{j \in G_{D}} \Psi^{p} d_{j}{ }^{p}}{\left|G_{T}\right|},
$$

and then, $\Theta_{j}$ is drawn uniformly from $[1 \bar{c}, 3 \bar{c}]$. The capacity of the emergency facility was obviously set to infinity. The disruption scenarios were randomly generated, such that each facility was possibly disrupted with probability $q \in\{0.01,0.03,0.05,0.1,0.15\}$, where $q$ gives the set of 
probabilies of facility disruptions. Several probabilities were intentionally chosen in order to present the effect of disruptions and the efficiency of the model, as disruption being an important factor. If duplicate scenarios were generated by this process, then the duplicates were withdrawn and the procedure was repeated till the $|S|$ unique scenarios were obtained.

\subsection{Performance of Algorithm}

Here, the efficiencies of SAA, and the proposed HHBSAA are compared. The efficiency of the proposed HHBSAA is analyzed the exact method and the SAA method over several sample sizes $(N)$ and number of samples $(M)$. For the SAA method, the test instances with $M=\{5,10,20\}$ and $N=\{5$, $10,20,30\}$ were experimented. On the other hand, all HHBSAA sample tests were set to $M=5$ with varying sample sizes from $N=\{5,10,15\}$. The results were reported according to 10 replications (i.e., 10 random sample instances for each combination of sample size and number of samples were generated) and solved with both SAA and HHBSAA. For $q \in\{0.01,0.03,0.05,0.1,0.15\}$, the comparison of the results of the two proposed algorithms is reported in Table 2. Both the results of best solution and various statistics of 10 replications are reported for each failure probability of failure.

The best solution obtained by each method throughout all replications was demonstrated as the $L_{\text {best }}$ column. For the test problem with $q=0.03$, the SAA's solution locates facilities $L_{\text {best }}=\{1,3,4$, 10 \} with $M=20$ and $N=30$, while the HHBSAA (for all sample sizes) and the exact solution method locate facilities $L_{\text {best }}=\{1,2,3,4,5,10\}$. The " $\xi_{\text {best }}$ " column illustrates the best obtained objective function value for the SAA, HHBSAA, and the exact method (i.e., $\xi^{\mathrm{SAA}}, \xi^{\mathrm{HHBSA}}$ and $\xi^{*}$ ). The $\xi^{\text {min }}$ and $\bar{\xi}^{M}$ columns are the minimum and average value of the sample optimal objective functions, respectively for the sample set related to the replication with the best objective. Here, two optimality gap measures are presented. Since the first gap $\left(G A P_{1}\right)$ according to the assumption is that $\bar{\xi}^{M}$ is a statistical lower bound on $\xi^{*}$, this gap is only suitable for SAA. It is defined as

$$
G A P_{1}=\frac{\xi^{S A A}-\bar{\xi}^{M}}{\bar{\xi}^{M}} \times 100 \%
$$

Another kind of optimality gap $\left(G A P_{2}\right)$ can be defined as

$$
G A P_{2}=\frac{\xi^{S A A}-\xi^{*}}{\xi^{*}} \times 100 \%
$$

Note that $G A P_{2}$ is suitable for both the SAA and the HHBSAA algorithms. Table 2 shows that once the scale of the test instance increases, the SAA's objective function is not constantly decreasing, whereas the CPU time is exponentially increasing. Correspondingly, increasing the sample size can lead to increase in the HHBSAA's CPU time; however the rate of the increase is less than that of the SAA. This is referred to the reduced number of HHBSAA iterations and increased efficiency of the algorithm. Anyhow, when the scale of the test instance for HHBSAA is increased beyond $N=20$, the CPU time starts to increase exponentially (it is like that of SAA). As the results in Table 1 demonstrate, the SAA algorithm cannot even find a feasible solution when $q$ increases ( $q>0.03$ ), while the HHBSAA algorithm can find suitable feasible solutions with reasonable optimal gaps at the reasonable times. 
In Table 1, with $q=0.01$, it can be concluded that the SAA method finds some feasible solutions when the value of $M$ and $N$ increase to more than 10 and 20 respectively, while the HHBSAA's solution is optimal even for the $(M, N)=\{(5,5)\}$. Since the effect of sampling is not significant with this probability of failure ( $q=0.01$ ), the RMCLNDP is comparably easy to solve. With $q=\{0.03,0.05\}$, the HHBSAA's average $\mathrm{GAP}_{2}$ is $0 \%$ with an average CPU time of $1113.5 \mathrm{~s}$ and $1696.78 \mathrm{~s}$, respectively. In comparison, the SAA's average $G A P_{2}$ is $10.42 \%$ and $5.10 \%$ with CPU time of 7131.66s and 10413.90s, respectively. Across all replications, SAA cannot find even any feasible solution (except for $q=0.03, M=20, N=30$ ) when the $M$ and the $N$ increase considerably, while HHBSAA can find the optimum solution for test instances $(M, N)=\{(5,5),(5,10)\}$ within much less CPU times.

Also, for $q=\{0.05,0.1,0.15\}$, Table 1 shows that SAA cannot find even any feasible solution, whereas HHBSAA finds the optimal solution in all of the test instances. With $q=0.1$ and in 4304.62s CPU time on the average, HHBSAA converged to the optimal solution with an optimality gap $\left(\mathrm{GAP}_{2}\right)$ of $8.54 \%$. The SAA's average CPU time is $12037.51 \mathrm{~s}$ without any feasible solution for all instances. With $q=0.15$ and in 5668.97s CPU time on the average, the HHBSAA converged to the optimum solution with an optimality gap $18.22 \%$. The SAA's average CPU time is 14325.59 s without any feasible solution for all the instances. The obtained results present that HHBSAA can find some feasible (and of course better quality) solutions in much less times than SAA.

Fig. 3, presents the impact of facility's failure probability on the efficiency of HHBSAA with $N=5$, 10 , and 20 in comparison with those of SAA with $N=5,10,20$, and 30 scenarios. It is remaindered that the CPU time performance of HHBSAA with $N=5,10$, and 20 is comparable to that of SAA with $N=20$ and significantly better than $N=30$. Hence, in comparison with SAA, HHBSAA not only find some feasible solutions, but also can improve the solution quality using an equivalent computational attempt. Moreover, the computing time needed for obtaining the same solution is much less with HHBSAA than SAA.

Fig. 4 demonstrates the impact of facility failure probability $(q)$ on the average solution quality $\left(\mathrm{GAP}_{2}\right)$ of HHBSAA with $N=5,10$, and 20 in comparison with those of SAA with $N=5,10,20$, and 30 scenarios. It is obviously concluded that HHBSAA is evermore outperforming SAA according to feasibility and quality of the obtained solution. It is noted that the values of "Gap " are not seen in Fig.4, because these values are more than 100, and thus are not presented in Fig. 4.

\section{Conclusions and Directions for Further Research}

In this study, the reliable capacitated logistic network design problem was investigated with a multi-configuration structure (RMCLNDP) in the presence of several system disruptions. Multiconfiguration structure includes multi-product, multi-vehicle, and multi-type link structure. Moreover, system disruptions include facility and transshipment link disruptions. The problem was formulated as a MILP model. It was proved that the proposed was an NP-hard problem. We also proposed an efficient hybrid heuristic algorithm. The proposed algorithm hybridization of SAA, the LP relaxation, and a two stage decomposing heuristic. The results of a detailed comprehensive computational analysis illustrated that the proposed algorithm substantially outperformed the integer programming approach in terms of both finding and verifying the efficient optimal (or near optimal) solutions at a reasonable processing times. Some directions for future research can be proposed as follows. Instead of scenario states, the system disruptions can be considered as some probability distributions and RMCLNDP can be reformulated accordingly. This may lead to a more accurate 
view of RMCLNDP and consequently to more practical solutions for the proposed problem. Another continuation of our work here is testing of other hybrid heuristic (meta-heuristic) or approximation algorithms in order to obtain more efficient solutions. This can be useful to decision makers in order to make more accurate and practical decisions on the proposed logistic networks. 
Reliable designing of capacitated logistics network

Table 2. Analyses of solution performance and CPU time efficiencies of SAA and HHBSAA for RMCLNDP with several facility failure probabilities $(q)$

\begin{tabular}{|c|c|c|c|c|c|c|c|c|c|c|}
\hline \multirow[t]{2}{*}{ Algorithm } & \multirow{2}{*}{$\mathrm{M}-\mathrm{N}$} & \multicolumn{9}{|l|}{$q=0.01$} \\
\hline & & $L_{\text {best }}$ & $\xi_{\text {best }}$ & $\xi^{\min }$ & $\bar{\xi}^{M}$ & $\sigma$ & $G A P_{1}$ & CPU-SAA (s) & Total CPU (s) & $G A P_{2}$ \\
\hline \multirow[t]{8}{*}{ SAA } & $5--5$ & $\mathrm{n} / \mathrm{a}$ & $\operatorname{Inf}$ & 86575.64 & 94192.79 & 4029.32 & $\mathrm{n} / \mathrm{a}$ & 62.54 & 445.13 & $\mathrm{n} / \mathrm{a}$ \\
\hline & $5--10$ & $\mathrm{n} / \mathrm{a}$ & Inf & 82995.39 & 90063.73 & 7733.73 & $\mathrm{n} / \mathrm{a}$ & 377.56 & 871.46 & $\mathrm{n} / \mathrm{a}$ \\
\hline & $5--20$ & $\mathrm{n} / \mathrm{a}$ & Inf & 84326.24 & 85757.58 & 835.94 & $\mathrm{n} / \mathrm{a}$ & 3426.15 & 4734.69 & $\mathrm{n} / \mathrm{a}$ \\
\hline & $5--30$ & $\mathrm{n} / \mathrm{a}$ & Inf & 83294.34 & 89645.94 & 1465.32 & $\mathrm{n} / \mathrm{a}$ & 5467.95 & 8004.25 & $\mathrm{n} / \mathrm{a}$ \\
\hline & $10--20$ & $1,4,10$ & 148452.65 & 84216.34 & 88916.45 & 2349.26 & 66.96 & 4351.64 & 5894.43 & 37.07 \\
\hline & $10--30$ & $1,3,10$ & 117345.61 & 82647.35 & 87342.61 & 5469.65 & 34.35 & 6431.26 & 9016.42 & 8.35 \\
\hline & $20--30$ & $1,3,10$ & 108302.34 & 83164.34 & 91469.24 & 8234.61 & 18.40 & 7841.25 & 10643.59 & 0.00 \\
\hline & \multicolumn{2}{|c|}{ Average } & 124700.20 & & & & 39.90 & & 5658.57 & 15.14 \\
\hline \multirow[t]{4}{*}{ HHBSAA } & $5--5$ & $1,3,10$ & 108302.34 & 86575.64 & 94192.79 & 4029.32 & 14.98 & 62.54 & 189.21 & 0.00 \\
\hline & $5--10$ & $1,3,10$ & 108302.34 & 82995.39 & 90063.73 & 7733.73 & 20.25 & 377.56 & 447.23 & 0.00 \\
\hline & $5--20$ & $1,3,10$ & 108302.34 & 84326.24 & 85757.58 & 835.94 & 26.29 & 3426.15 & 2704.06 & 0.00 \\
\hline & \multicolumn{2}{|c|}{ Average } & 108302.34 & & & & 20.51 & & 1113.50 & 0.00 \\
\hline Exact & - & $1,3,10$ & 108302.34 & - & - & - & - & - & 12049.25 & - \\
\hline \multirow[t]{2}{*}{ Algorithm } & \multirow{2}{*}{ M-N } & $q=0.03$ & & & & & & & & \\
\hline & & $L_{\text {best }}$ & $\xi_{\text {best }}$ & $\xi^{\min }$ & $\bar{\xi}^{M}$ & $\sigma$ & $G A P_{1}$ & CPU-SAA (s) & Total CPU (s) & $G A P_{2}$ \\
\hline \multirow[t]{6}{*}{ SAA } & $5--5$ & $\mathrm{n} / \mathrm{a}$ & $\overline{\operatorname{Inf}}$ & 96806.47 & 135375.19 & 32619.79 & $\mathrm{n} / \mathrm{a}$ & 102.43 & 812.45 & $\mathrm{n} / \mathrm{a}$ \\
\hline & $5--10$ & $\mathrm{n} / \mathrm{a}$ & Inf & 84785.32 & 161420.13 & 56205.30 & $\mathrm{n} / \mathrm{a}$ & 589.47 & 1234.64 & $\mathrm{n} / \mathrm{a}$ \\
\hline & $5--20$ & $\mathrm{n} / \mathrm{a}$ & $\operatorname{Inf}$ & 102765.35 & 125097.69 & 13183.93 & $\mathrm{n} / \mathrm{a}$ & 3573.54 & 5879.34 & $\mathrm{n} / \mathrm{a}$ \\
\hline & $5--30$ & $\mathrm{n} / \mathrm{a}$ & Inf & 115642.31 & 136947.62 & 8654.91 & $\mathrm{n} / \mathrm{a}$ & 6245.14 & 9321.64 & $\mathrm{n} / \mathrm{a}$ \\
\hline & $10--20$ & $\mathrm{n} / \mathrm{a}$ & $\operatorname{Inf}$ & 120624.37 & 154624.61 & 9451.62 & $\mathrm{n} / \mathrm{a}$ & 5876.45 & 8024.56 & $\mathrm{n} / \mathrm{a}$ \\
\hline & $10--30$ & $\mathrm{n} / \mathrm{a}$ & Inf & 133987.62 & 165487.62 & 10624.62 & $\mathrm{n} / \mathrm{a}$ & 7241.64 & 10974.34 & $\mathrm{n} / \mathrm{a}$ \\
\hline
\end{tabular}


Table 2. Continued.

\begin{tabular}{|c|c|c|c|c|c|c|c|c|c|c|}
\hline & $20--30$ & $1,3,4,10$ & 286648.32 & 145624.31 & 176947.62 & 9978.64 & 62.00 & 8468.32 & 13674.67 & 10.42 \\
\hline & & rerage & $\mathrm{n} / \mathrm{a}$ & & & & $\mathrm{n} / \mathrm{a}$ & & 7131.66 & 10.42 \\
\hline \multirow[t]{4}{*}{ HHBSAA } & $5--5$ & $1,2,3,4,5,10$ & 259603.27 & 96806.47 & 135375.19 & 32619.79 & 91.77 & 102.43 & 576.67 & 0.00 \\
\hline & $5--10$ & $1,2,3,4,5,10$ & 259603.27 & 84785.32 & 161420.13 & 56205.30 & 60.82 & 589.47 & 809.35 & 0.00 \\
\hline & $5--20$ & $1,2,3,4,5,10$ & 259603.27 & 102765.35 & 125097.69 & 13183.93 & 107.52 & 3573.54 & 3704.32 & 0.00 \\
\hline & \multicolumn{2}{|c|}{ Average } & 259603.27 & & & & 86.70 & & 1696.78 & 0.00 \\
\hline Exact & - & $1,2,3,4,5,10$ & 259603.27 & - & - & - & - & - & 13649.24 & - \\
\hline \multirow[t]{2}{*}{ Algorithm } & \multirow{2}{*}{$\mathrm{M}-\mathrm{N}$} & $q=0.05$ & & & & & & & & \\
\hline & & $L_{\text {best }}$ & $\xi_{\text {best }}$ & $\xi^{\min }$ & $\bar{\xi}^{M}$ & $\sigma$ & $G A P_{1}$ & CPU-SAA (s) & Total CPU (s) & $G A P_{2}$ \\
\hline \multirow[t]{8}{*}{ SAA } & $5--5$ & $\mathrm{n} / \mathrm{a}$ & Inf & 98075.96 & 105318.57 & 6180.92 & $\mathrm{n} / \mathrm{a}$ & 115.14 & 1043.14 & $\mathrm{n} / \mathrm{a}$ \\
\hline & $5--10$ & $\mathrm{n} / \mathrm{a}$ & $\operatorname{Inf}$ & 118738.26 & 138246.13 & 13648.06 & $\mathrm{n} / \mathrm{a}$ & 764.23 & 1384.62 & $\mathrm{n} / \mathrm{a}$ \\
\hline & $5--20$ & $\mathrm{n} / \mathrm{a}$ & $\operatorname{Inf}$ & 132478.64 & 144036.13 & 7399.73 & $\mathrm{n} / \mathrm{a}$ & 4562.41 & 6747.58 & $\mathrm{n} / \mathrm{a}$ \\
\hline & $5--30$ & $\mathrm{n} / \mathrm{a}$ & $\operatorname{Inf}$ & 137845.64 & 149967.51 & 8671.68 & $\mathrm{n} / \mathrm{a}$ & 8265.24 & 12079.48 & $\mathrm{n} / \mathrm{a}$ \\
\hline & $10--20$ & $\mathrm{n} / \mathrm{a}$ & Inf & 137194.35 & 149448.34 & 7942.34 & $\mathrm{n} / \mathrm{a}$ & 7465.24 & 11846.82 & $\mathrm{n} / \mathrm{a}$ \\
\hline & $10--30$ & $\mathrm{n} / \mathrm{a}$ & Inf & 147473.95 & 153178.69 & 6947.62 & $\mathrm{n} / \mathrm{a}$ & 9194.00 & 16946.31 & $\mathrm{n} / \mathrm{a}$ \\
\hline & $20--30$ & $\mathrm{n} / \mathrm{a}$ & Inf & 150447.64 & 159448.35 & 8164.35 & $\mathrm{n} / \mathrm{a}$ & 11467.28 & 22849.36 & $\mathrm{n} / \mathrm{a}$ \\
\hline & \multicolumn{2}{|c|}{ Average } & $\mathrm{n} / \mathrm{a}$ & & & & $\mathrm{n} / \mathrm{a}$ & & 10413.90 & $\mathrm{n} / \mathrm{a}$ \\
\hline \multirow[t]{4}{*}{ HHBSAA } & $5--5$ & $1,3,4,10$ & 405649.64 & 98075.96 & 105318.57 & 6180.92 & 285.16 & 115.14 & 872.49 & 10.05 \\
\hline & $5--10$ & $1,2,3,10$ & 387947.32 & 118738.26 & 138246.13 & 13648.06 & 180.62 & 764.23 & 1206.14 & 5.25 \\
\hline & $5--20$ & $1,2,3,6,10$ & 368596.28 & 132478.64 & 144036.13 & 7399.73 & 155.91 & 4562.41 & 4945.24 & 0.00 \\
\hline & \multicolumn{2}{|c|}{ Average } & 387397.75 & & & & 207.23 & & 2341.29 & 5.10 \\
\hline Exact & - & $1,2,3,6,10$ & 368596.28 & - & - & - & - & - & 16437.62 & - \\
\hline
\end{tabular}


Reliable designing of capacitated logistics network

Table 2. Continued

\begin{tabular}{|c|c|c|c|c|c|c|c|c|c|c|}
\hline \multirow[t]{2}{*}{ Algorithm } & \multirow{2}{*}{$\mathrm{M}-\mathrm{N}$} & \multicolumn{9}{|l|}{$q=0.1$} \\
\hline & & $L_{\text {best }}$ & $\xi_{\text {best }}$ & $\xi^{\min }$ & $\bar{\xi}^{M}$ & $\sigma$ & $G A P_{1}$ & CPU-SAA (s) & Total CPU (s) & $G A P_{2}$ \\
\hline \multirow[t]{8}{*}{ SAA } & $5--5$ & $\mathrm{n} / \mathrm{a}$ & Inf & 122346.61 & 126497.54 & 9432.81 & $\mathrm{n} / \mathrm{a}$ & 162.64 & 1456.71 & $\mathrm{n} / \mathrm{a}$ \\
\hline & $5--10$ & $\mathrm{n} / \mathrm{a}$ & Inf & 126947.34 & 131647.29 & 8794.36 & $\mathrm{n} / \mathrm{a}$ & 1102.37 & 2697.62 & $\mathrm{n} / \mathrm{a}$ \\
\hline & $5--20$ & $\mathrm{n} / \mathrm{a}$ & $\operatorname{Inf}$ & 128649.67 & 134957.49 & 7649.26 & $\mathrm{n} / \mathrm{a}$ & 5064.36 & 9649.34 & $\mathrm{n} / \mathrm{a}$ \\
\hline & $5--30$ & $\mathrm{n} / \mathrm{a}$ & Inf & 137697.29 & 145649.57 & 10452.72 & $\mathrm{n} / \mathrm{a}$ & 9226.42 & 14645.91 & $\mathrm{n} / \mathrm{a}$ \\
\hline & $10--20$ & $\mathrm{n} / \mathrm{a}$ & Inf & 142947.82 & 149649.64 & 8465.36 & $\mathrm{n} / \mathrm{a}$ & 8194.63 & 12346.25 & $\mathrm{n} / \mathrm{a}$ \\
\hline & $10--30$ & $\mathrm{n} / \mathrm{a}$ & Inf & 152649.76 & 158935.62 & 8064.92 & $\mathrm{n} / \mathrm{a}$ & 10064.53 & 18423.14 & $\mathrm{n} / \mathrm{a}$ \\
\hline & $20--30$ & $\mathrm{n} / \mathrm{a}$ & Inf & 161697.83 & 168346.34 & 10312.62 & $\mathrm{n} / \mathrm{a}$ & 12764.26 & 25043.59 & $\mathrm{n} / \mathrm{a}$ \\
\hline & \multicolumn{2}{|c|}{ Average } & $\mathrm{n} / \mathrm{a}$ & & & & $\mathrm{n} / \mathrm{a}$ & & 12037.51 & $\mathrm{n} / \mathrm{a}$ \\
\hline \multirow[t]{5}{*}{ HHBSAA } & $5--5$ & $1,3,4,10$ & 497643.69 & 122346.61 & 126497.54 & 9432.81 & 293.40 & 162.64 & 1154.61 & 16.91 \\
\hline & $5--10$ & $1,3,5,10$ & 467943.16 & 126947.34 & 131647.29 & 8794.36 & 255.45 & 1102.37 & 2006.52 & 9.94 \\
\hline & $5--20$ & $1,2,3,10$ & 445643.72 & 128649.67 & 134957.49 & 7649.26 & 230.21 & 5064.36 & 6243.91 & 4.70 \\
\hline & $5--30$ & $1,2,3,5,6,10$ & 436749.34 & 132697.29 & 140649.57 & 10452.72 & 210.52 & 9226.42 & 7814.62 & 2.61 \\
\hline & \multicolumn{2}{|c|}{ Average } & 461994.98 & & & & 247.40 & & 4304.92 & 8.54 \\
\hline Exact & - & $1,2,3,6,7,10$ & 425649.76 & - & - & - & - & - & 20037.62 & - \\
\hline \multirow[t]{2}{*}{ Algorithm } & \multirow{2}{*}{ M-N } & $q=0.15$ & & & & & & & & \\
\hline & & $L_{\text {best }}$ & $\xi_{\text {best }}$ & $\xi \min$ & $\bar{\xi}^{M}$ & $\sigma$ & $G A P_{1}$ & CPU-SAA (s) & Total CPU (s) & $G A P_{2}$ \\
\hline \multirow[t]{5}{*}{ SAA } & $5--5$ & $\mathrm{n} / \mathrm{a}$ & Inf & 129946.35 & 133647.54 & 6784.24 & $\mathrm{n} / \mathrm{a}$ & 181.64 & 1614.31 & $\mathrm{n} / \mathrm{a}$ \\
\hline & $5--10$ & $\mathrm{n} / \mathrm{a}$ & Inf & 134647.84 & 138456.05 & 4352.16 & $\mathrm{n} / \mathrm{a}$ & 1615.01 & 3314.24 & $\mathrm{n} / \mathrm{a}$ \\
\hline & $5--20$ & $\mathrm{n} / \mathrm{a}$ & Inf & 141947.52 & 145346.47 & 8345.25 & $\mathrm{n} / \mathrm{a}$ & 8674.15 & 11456.71 & $\mathrm{n} / \mathrm{a}$ \\
\hline & $5--30$ & $\mathrm{n} / \mathrm{a}$ & $\operatorname{Inf}$ & 15079.14 & 155974.29 & 9745.64 & $\mathrm{n} / \mathrm{a}$ & 10593.16 & 15435.46 & $\mathrm{n} / \mathrm{a}$ \\
\hline & $10--20$ & $\mathrm{n} / \mathrm{a}$ & $\operatorname{Inf}$ & 149003.54 & 154364.24 & 7943.24 & $\mathrm{n} / \mathrm{a}$ & 9945.14 & 16972.64 & $\mathrm{n} / \mathrm{a}$ \\
\hline
\end{tabular}


Table 2. Continued.

\begin{tabular}{|c|c|c|c|c|c|c|c|c|c|}
\hline 10--30 & $\mathrm{n} / \mathrm{a}$ & Inf & 163874.36 & 168792.04 & 8146.21 & $\mathrm{n} / \mathrm{a}$ & 11746.15 & 23006.34 & $\mathrm{n} / \mathrm{a}$ \\
\hline 20--30 & $\mathrm{n} / \mathrm{a}$ & Inf & 170467.16 & 175924.19 & 8879.34 & $\mathrm{n} / \mathrm{a}$ & 13456.12 & 28479.43 & $\mathrm{n} / \mathrm{a}$ \\
\hline & Average & $\mathrm{n} / \mathrm{a}$ & & & & $\mathrm{n} / \mathrm{a}$ & & 14325.59 & $\mathrm{n} / \mathrm{a}$ \\
\hline $5--5$ & $1,4,5,10$ & 678462.34 & 129946.35 & 133647.54 & 6784.24 & 407.65 & 181.64 & 1307.46 & 34.44 \\
\hline $5--10$ & $1,4,5,10$ & 614325.71 & 134647.84 & 138456.05 & 4352.16 & 343.70 & 1615.01 & 2876.45 & 21.73 \\
\hline $5--20$ & $1,2,3,7,8,10$ & 572345.16 & 141947.52 & 145346.47 & 8345.25 & 293.78 & 6974.15 & 8246.34 & 13.41 \\
\hline $5--30$ & $1,2,3,5,7,10$ & 521345.13 & 15079.14 & 155974.29 & 9745.64 & 234.25 & 5793.16 & 10245.64 & 3.31 \\
\hline & Average & 596619.59 & & & & 319.84 & & 5668.97 & 18.22 \\
\hline - & $1,2,3,5,7,10$ & 504649.76 & - & - & - & - & - & 24615.31 & - \\
\hline
\end{tabular}




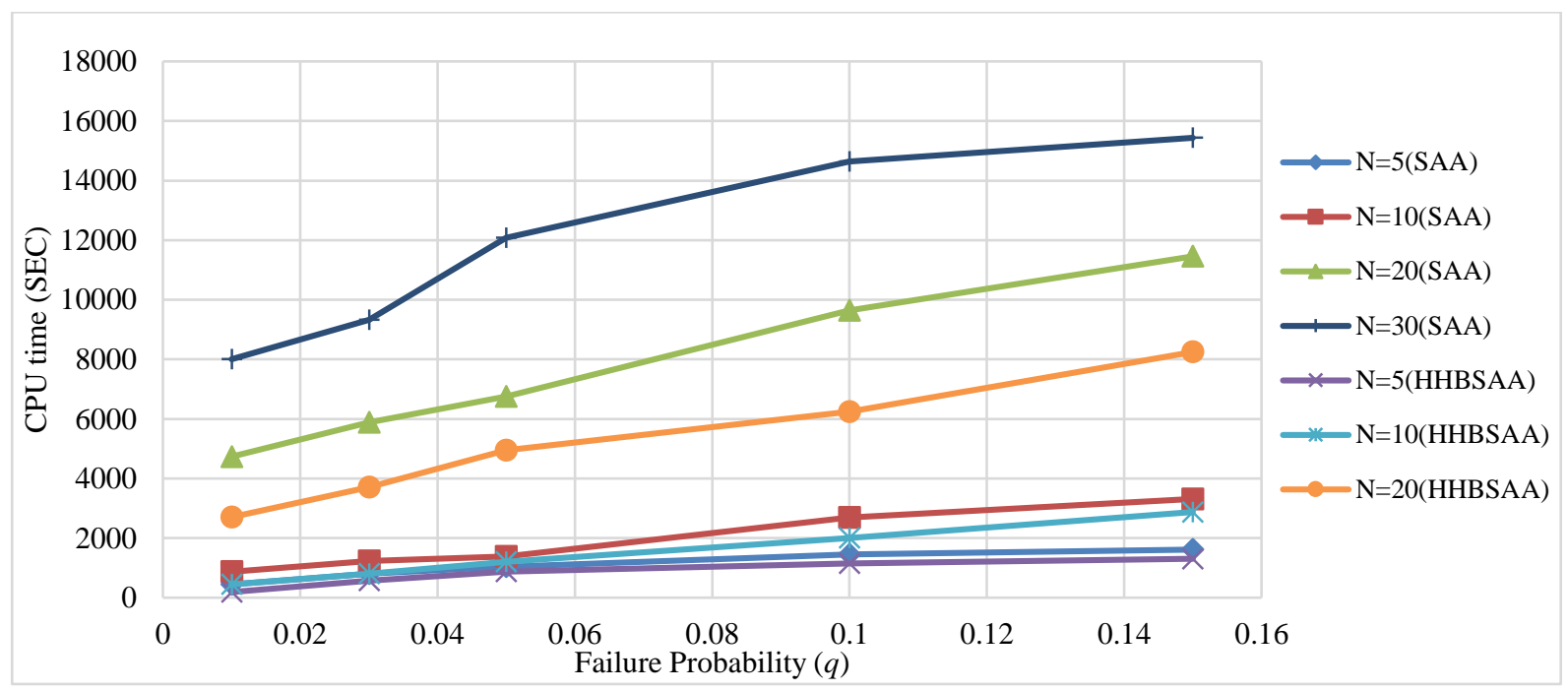

Figure. 4. Comparison of HHBSAA and SAA with several test instance sizes $(N)$ and failure probabilities $(q)$ regarding the CPU times

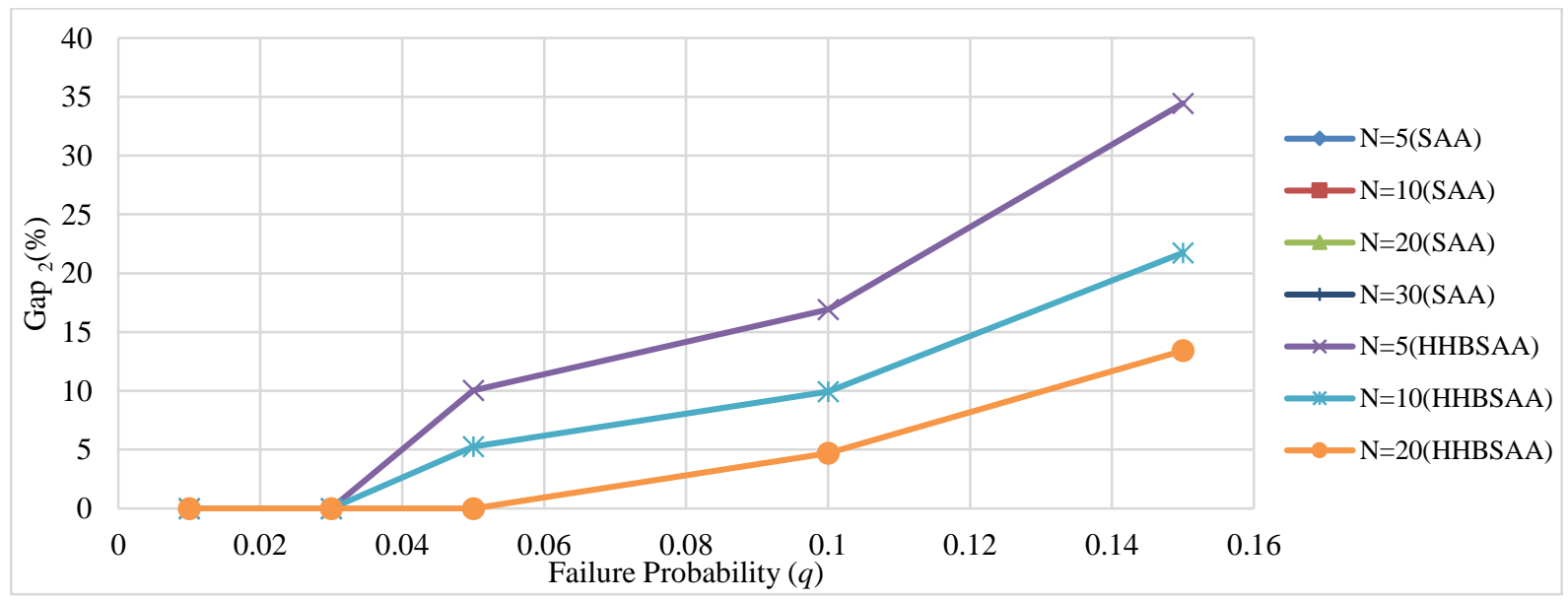

Figure. 4. Comparison of HHBSAA and SAA with several test instance sizes $(N)$ and failure probabilities $(q)$ regarding solution quality as optimality gap $\left(\mathrm{GAP}_{2}\right)$

\section{References}

[1] Aboolian, R., Cui, T., and Shen, Z.-J. M. (2012), An Efficient Approach for Solving Reliable Facility Location Models, INFORMS Journal on Computing, 25(4), 720-729.

[2] Ahmed, S., and Shapiro, A. (2002), The sample average approximation method for stochastic programs with integer recourse, Optimization Online, http://www.optimizationonline.org/S.

[3] Amrani, H., Martel, A., Zufferey, N., and Makeeva, P. (2011), A variable neighborhood search heuristic for the design of multicommodity production-distribution networks with alternative facility configurations, OR Spectrum, 33(4), 989-1007. 
[4] Aydin, N., and Murat, A. (2013), A swarm intelligence based sample average approximation algorithm for the capacitated reliable facility location problem, International Journal of Production Economics, 145(1), 173-183.

[5] Azizi, N., Chauhan, S., Salhi, S., and Vidyarthi, N. (2014), The impact of hub failure in hub-and-spoke networks: Mathematical formulations and solution techniques, Computers \& Operations Research, 65, 174-188.

[6] Badri, H., Bashiri, M., and Hejazi, T. H. (2013), Integrated strategic and tactical planning in a supply chain network design with a heuristic solution method, Computers \& Operations Research, 40(4), 1143-1154.

[7] Bashiri, M., Badri, H., and Talebi, J. (2012), A new approach to tactical and strategic planning in production-distribution networks, Applied Mathematical Modelling, 36(4), 1703-1717.

[8] Berman, O., Krass, D., and Menezes, M. B. C. (2007), Facility reliability issues in network p-median problems: strategic centralization and co-location effects, Operations Research, 55(2), 332-350.

[9] Berman, O., Krass, D., and Menezes, M. B. C. (2010), Location problems with two unreliable facilities on a line allowing correlated failures, Working Paper, Rotman School of Management, University of Toronto, Toronto, Ontario, Canada.

[10] Cardona-Valdes, Y., Alvarez, A., and Pacheco, J. (2014), Metaheuristic procedure for a biobjective supply chain design problem with uncertainty, Transportation Research Part B: Methodological, 60, 66-84.

[11] Chen, C.-L., and Lee, W.-C. (2004), Multi-objective optimization of multi-echelon supply chain networks with uncertain product demands and prices, Computers \& Chemical Engineering, 28(6), 1131-1144.

[12] Cui, T., Ouyang, Y., and Shen, Z. J. M. (2010), Reliable facility location design under the risk of disruptions, Operations Research, 58(4-Part-1), 998-1011.

[13] Daskin, M. S., Snyder, L. V., and Berger, R. T. (2005), Facility location in supply chain design, Logistics Systems: Design and Optimization, Springer, 39-65.

[14] Drezner, Z. (1987), Heuristic solution methods for two location problems with unreliable facilities, Journal of the Operational Research Society, 38(6), 509-514.

[15] Duan, Q., and Liao, T. W. (2013), Optimization of replenishment policies for decentralized and centralized capacitated supply chains under various demands, International Journal of Production Economics, 142(1), 194-204.

[16] El-Sayed, M., Afia, N., and El-Kharbotly, A. (2010), A stochastic model for forwardreverse logistics network design under risk, Computers \& Industrial Engineering, 58(3), 423-431.

[17] Esmaeilikia, M., Fahimnia, B., Sarkis, J., Govindan, K., Kumar, A., and Mo, J. (2014), Tactical supply chain planning models with inherent flexibility: definition and review, Annals of Operations Research, 244(2), 407-427.

[18] Fattahi, M., Mahootchi, M., Moattar Husseini, S. M., Keyvanshokooh, E., and Alborzi, F. (2015), Investigating replenishment policies for centralised and decentralised supply chains using stochastic programming approach, International Journal of Production Research, 53(1), 41-69.

[19] Ferrio, J., and Wassick, J. (2008), Chemical supply chain network optimization, Computers \& Chemical Engineering, 32(11), 2481-2504.

[20] Ferris, M. C., and Ruszczynski, A. (2000), Robust path choice in networks with failures, Networks, 35,200-18.

[21] Garcia-Herreros, P., Wassick, J. M., and Grossmann, I. E. (2014), Design of resilient supply chains with risk of facility disruptions, Industrial \& Engineering Chemistry Research, 53(44), 17240-17251. 
[22] Haldar, A., Ray, A., Banerjee, D., and Ghosh, S. (2014), Resilient supplier selection under a fuzzy environment, International Journal of Management Science and Engineering Management, 9(2), 147-156.

[23] Hatefi, S. M., Jolai, F., Torabi, S. A., and Tavakkoli-Moghaddam, R. (2014), A credibilityconstrained programming for reliable forward-reverse logistics network design under uncertainty and facility disruptions, International Journal of Computer Integrated Manufacturing, 28(6), 664-678.

[24] Hennet, J.-C., and Arda, Y. (2008), Supply chain coordination: A game-theory approach, Engineering Applications of Artificial Intelligence, 21(3), 399-405.

[25] Ivanov, D., Pavlov, A., and Sokolov, B. (2014), Optimal distribution (re) planning in a centralized multi-stage supply network under conditions of the ripple effect and structure dynamics, European Journal of Operational Research, 237(2), 758-770.

[26] Jabbarzadeh, A., Jalali Naini, S. G., Davoudpour, H., and Azad, N. (2012), Designing a Supply Chain Network under the Risk of Disruptions, Mathematical Problems in Engineering, 12 (12), 1-23.

[27] Jamshidi, R., Fatemi Ghomi, S. M. T., and Karimi, B. (2012), Multi-objective green supply chain optimization with a new hybrid memetic algorithm using the Taguchi method, Scientia Iranica, 19(6), 1876-1886.

[28] Jemai, Z., and Karaesmen, F. (2007), Decentralized inventory control in a two-stage capacitated supply chain, IIE Transactions, 39(5), 501-512.

[29] Karimi-Nasab, M., Shishebori, D., and Jalali-Naini, S. G. R. (2013), Multi-objective optimisation for pricing and distribution in a supply chain with stochastic demands, International Journal of Industrial and Systems Engineering, 13(1), 56-72.

[30] Keyvanshokooh, E., Fattahi, M., Seyed-Hosseini, S. M., and Tavakkoli-Moghaddam, R. (2013), A dynamic pricing approach for returned products in integrated forward/reverse logistics network design, Applied Mathematical Modelling, 37(24), 10182-10202.

[31] Keyvanshokooh, E., Ryan, S. M., and Kabir, E. (2016), Hybrid robust and stochastic optimization for closed-loop supply chain network design using accelerated Benders decomposition, European Journal of Operational Research, 249(1), 76-92.

[32] Kleywegt, A. J., Shapiro, A., and Homem-de-Mello, T. (2002), The sample average approximation method for stochastic discrete optimization, SIAM Journal on Optimization, 12(2), 479-502.

[33] Klibi, W., Martel, A., and Guitouni, A. (2009), The design of robust value-creating supply chain networks: a critical review, European Journal of Operational Research, 203(2), 283 293.

[34] Liberatore, F., Scaparra, M. P., and Daskin, M. S. (2012), Hedging against disruptions with ripple effects in location analysis, Omega, 40(1), 21-30.

[35] Lim, M., Daskin, M.S., Bassamboo, A., and Chopra, S. (2009), Facility location decisions in supply chain networks with random disruption and imperfect information, Working paper, Department of Business Administration, University of Illinois.

[36] Mahajan, J., Radas, S., and Vakharia, A. J. (2002), Channel Strategies and Stocking Policies in Uncapacitated and Capacitated Supply Chains, Decision Sciences, 33(2), 191-222.

[37] Meixell, M. J., and Gargeya, V. B. (2005), Global supply chain design: A literature review and critique, Transportation Research Part E: Logistics and Transportation Review, 41(6), 531-550.

[38] Mirzapour Al-E-Hashem, S. M. J., Malekly, H., and Aryanezhad, M. B. (2011), A multiobjective robust optimization model for multi-product multi-site aggregate production planning in a supply chain under uncertainty, International Journal of Production Economics, 134(1), 28-42. 
[39] Nepal, B., Murat, A., and Babu Chinnam, R. (2012), The bullwhip effect in capacitated supply chains with consideration for product life-cycle aspects, International Journal of Production Economics, 136(2), 318-331.

[40] O’Hanley, J. R., and Church, R. L. (2011), Designing robust coverage networks to hedge against worst-case facility losses, European Journal of Operational Research, 209(1), 2336.

[41] Park, B. J., Choi, H. R., and Kang, M. H. (2007), Integration of production and distribution planning using a genetic algorithm in supply chain management, Analysis and Design of Intelligent Systems using Soft Computing Techniques, Springer, 416-426.

[42] Pasandideh, S. H. R., Niaki, S. T. A., and Asadi, K. (2014), Bi-objective optimization of a multi-product multi-period three-echelon supply chain problem under uncertain environments: NSGA-II and NRGA, Information Sciences, 292, 57-74.

[43] Peng, P., Snyder, L. V., Lim, A., and Liu, Z. (2011), Reliable logistics networks design with facility disruptions, Transportation Research Part B: Methodological, 45(8), 1190-1211.

[44] Rahmaniani R and Ghaderi A (2015), An algorithm with different exploration mechanisms: experimental results to capacitated facility location/network design problem, Expert Systems with Applications 42(7): 3790-3800.

[45] Sarrafha, K., Rahmati, S. H. A., Niaki, S. T. A., and Zaretalab, A. Z. (2014), A bi-objective integrated procurement, production, and distribution problem of a multi-echelon supply chain network design: A new tuned MOEA, Computers \& Operations Research, 54, 35-51.

[46] Schutz, P., Tomasgard, A., and Ahmed, S. (2009), Supply chain design under uncertainty using sample average approximation and dual decomposition, European Journal of Operational Research, 199(2), 409-419.

[47] Sever, D., Dellaert, N., van Woensel, T., and de Kok, T. (2013), Dynamic shortest path problems: Hybrid routing policies considering network disruptions, Computers \& Operations Research, 40(12), 2852-2863.

[48] Shishebori, D., Jabalameli, M. S., and Jabbarzadeh, A. (2013), Facility location-network design problem: reliability and investment budget constraint, Journal of Urban Planning and Development, 140(3), 04014005.

[49] Shishebori, D., and Jabalameli, M. S. (2013a), Improving the efficiency of medical services systems: a new integrated mathematical modeling approach, Mathematical Problems in Engineering, 1: 1-13.

[50] Shishebori, D., and Jabalameli, M. S. (2013b), A new integrated mathematical model for optimizing facility location and network design policies with facility disruptions, Life Science Journal, 10(1), 1896-1906.

[51] Shishebori, D., Snyder, L. V., and Jabalameli, M. S. (2014), A reliable budget- constrained facility location/network design problem with unreliable facilities, Networks and Spatial Economics, 14(3-4), 549-580.

[52] Shishebori D., and Yousefi Babadi A. (2015), Robust and reliable medical services network design underuncertain environment and system disruptions, Transportation Research Part E 77: 268-288.

[53] Sitompul, C., Aghezzaf, E.-H., Dullaert, W., and Landeghem, H. V. (2008), Safety stock placement problem in capacitated supply chains, International Journal of Production Research, 46(17), 4709-4727.

[54] Snyder, L. V. (2003), Supply chain robustness and reliability: Models and algorithms (PHD Thesis), Northwestern University, Evanston, IL.

[55] Snyder, L. V., Atan, Z., Peng, P., Rong, Y., Schmitt, A. J., and Sinsoysal, B. (2012), OR/MS models for supply chain disruptions: A review, SSRN eLibrary.

[56] Snyder, L. V., and Daskin, M. S. (2005), Reliability models for facility location: The expected failure cost case, Transportation Science, 39(3), 400-416. 
[57] Snyder, L. V., Scaparra, M. P., Daskin, M. S., and Church, R. L. (2006), Planning for disruptions in supply chain networks, Tutorials in operations research, 2, 234-257.

[58] Snyder, L. V., and Daskin, M. S. (2007), Models for reliable supply chain network design. Critical Infrastructure : Reliability and Vulnerability, A.T. Murray and T.H. Grubesic (eds.), , Chapter 13, 257-289.

[59] Song, D.-P., Dong, J.-X., and Xu, J. (2014), Integrated inventory management and supplier base reduction in a supply chain with multiple uncertainties, European Journal of Operational Research, 232(3), 522-536.

[60] Soni, S., and Pirkul, H. (2000), Design of survivable networks with connectivity requirements, Telecommunication Systems 20 (1-2), 133-149.

[61] Toktas-Palut, P., and Fusun., U. (2011), Coordination in a two-stage capacitated supply chain with multiple suppliers, European Journal of Operational Research, 212(1), 43-53.

[62] Vahdani, B., Tavakkoli-Moghaddam, R., Modarres, M., and Baboli, A. (2012), Reliable design of a forward/reverse logistics network under uncertainty: a robust-M/M/c queuing model, Transportation Research Part E: Logistics and Transportation Review, 48(6), 11521168.

[63] Vahdani, B., Tavakkoli-Moghaddam, R., Jolai, F., and Baboli, A. (2013), Reliable design of a closed loop supply chain network under uncertainty: An interval fuzzy possibilistic chance-constrained model, Engineering Optimization, 45(6), 745-765.

[64] Vahdani, B., Tavakkoli-Moghaddam, R., and Jolai, F. (2013), Reliable design of a logistics network under uncertainty: A fuzzy possibilistic-queuing model, Applied Mathematical Modelling, 37(5), 3254-3268.

[65] Vahdani, B., Dehbari, S., Naderi-Beni, M., and Zeinali, E. (2014), An artificial intelligence approach for fuzzy possibilistic-stochastic multi-objective logistics network design, Neural Computing and Applications, 25(7-8), 1887-1902.

[66] Vahdani, B., Razmi, J., and Tavakkoli-Moghaddam, R. (2012), Fuzzy possibilistic modeling for closed loop recycling collection networks, Environmental Modeling \& Assessment, 17(6), 623-637.

[67] Vahdani, B., (2015), An optimization model for multi-objective closed-loop supply chain network under uncertainty: a hybrid fuzzy-stochastic programming method, Iranian Journal of Fuzzy Systems, 12, 33-57.

[68] Vahdani, B., and Mohammadi, M. (2015), A bi-objective interval-stochastic robust optimization model for designing closed loop supply chain network with multi-priority queuing system, International Journal of Production Economics, 170, 67-87.

[69] Waller, S. T., Ziliaskopoulos, A.K. (2002), On the online shortest path problem with limited arc cost dependencies, Networks, 40(4), 216-27.

[70] You, F., and Grossmann, I. E. (2008), Design of responsive supply chains under demand uncertainty, Computers \& Chemical Engineering, 32(12), 3090-3111. 